\title{
LASERS AND OPTICS FOR A GAMMA-GAMMA COLLIDER
}

\author{
J. Gronberg, D. Asner, S. Boege, J. Early, K. Skulina, K. van Bibber, \\ LLNL, Livermore, CA 94550, USA * \\ T. Markiewicz, SLAC, Stanford, CA 94025, USA ${ }^{\dagger}$
}

\begin{abstract}
The production of high energy photon colliders through Compton back scattering of laser photons was proposed in the early 1980s [1]. Recent developments in high average power short pulse lasers have made such a collider feasible. The Lawrence Livermore Lab has embarked on a multi disciplinary effort to demonstrate a working design of a photon collider. Such a design requires the participation of experts in lasers, optics, accelerators and physics simulation. A laser design, based on the MERCURY architecture, that can provide the necessary laser power is reported along with the design of focusing optics able to be integrated into the confined space of a detector. Having demonstrated the technical feasibility of a photon collider, the expected physics reach of the benchmark $\gamma \gamma \rightarrow \mathrm{H}^{0}$ mode in our preliminary collider design is reported.
\end{abstract}

\section{INTRODUCTION}

The scientific advantages of incorporating high-energy $\gamma \gamma$ collisions into an $e^{+} e^{-}$collider design have been discussed from the earliest collider physics meeting in Saariselka, Findland in 1989. The loop diagram in $\gamma \gamma \rightarrow$ Higgs opens a window on new physics beyond the standard model, since the loop receives contributions from all particles which have charge and mass. However, the technical challenges posed by the laser system and the interaction region (IR) seemed to be overwhelming and have kept the option of a $\gamma \gamma$ collider out of the mainstream collider program. There has been sufficient recent progress on both these fronts to change that view and to allow the $\gamma \gamma$ collider to be considered as a feasible addition to a Linear Collider project.

\section{COMPTON BACKSCATTERING}

Compton backscattering is a two-body QED process whereby a low energy $(1 \mathrm{eV})$ laser photon interacts with a high energy electron to produce a high energy photon. The high energy photon tends to follow the direction of the electron. Therefore, if the conversion happens a short distance from the interaction point (IP) of an $e^{+} e^{-}$collider, a comparable luminosity of $\gamma \gamma$ collisions will be produced.

\footnotetext{
* This work was performed under the auspices of the U.S. Department of Energy by the University of California, Lawrence Livermore national Laboratory under Contract No. W-7405-Eng-48.

$\dagger$ Work performed under the auspices of the U.S. Department of Energy under Contract No. DE-AC03-76-SF00515.
}

The calculation of this process is reported in detail in [2] and incorporated into the CAIN Monte Carlo program [3].

In order to achieve a high luminosity of $\gamma \gamma$ collisions every electron bunch in the collider must be met with a high power laser pulse. This requires a laser architecture capable of high average power and capable of producing pulses matched to the time format of electron bunches in the accelerator. The laser power required is minimized when the laser pulse duration is matched to the electron bunch length of 100 microns. This argues for a laser pulse duration of about 1.8 ps FWHM. For that pulse duration and assuming that the laser can be focused to a diffraction limited spot we find that each laser pulse must have an energy of 1 Joule so that $63 \%$ of the electrons in a bunch will Compton backscatter. Increasing the energy past this point results in diminishing returns as one asymptotically approaches $100 \%$ conversion.

While the high energy photons tend to follow the direction of the initial electron, they receive an additional divergence of order 1 microradian from the Compton backscattering process. This implies that the conversion must occur within millimeters of the IP to prevent this spread from degrading the achievable spot size. This requires that the optics used to focus the laser pulses be co-located in the confined space of the interaction region. A design for such an optical system must not only have good optical properties but it must also not interfere with the operation of the accelerator or degrade the performance of the detector.

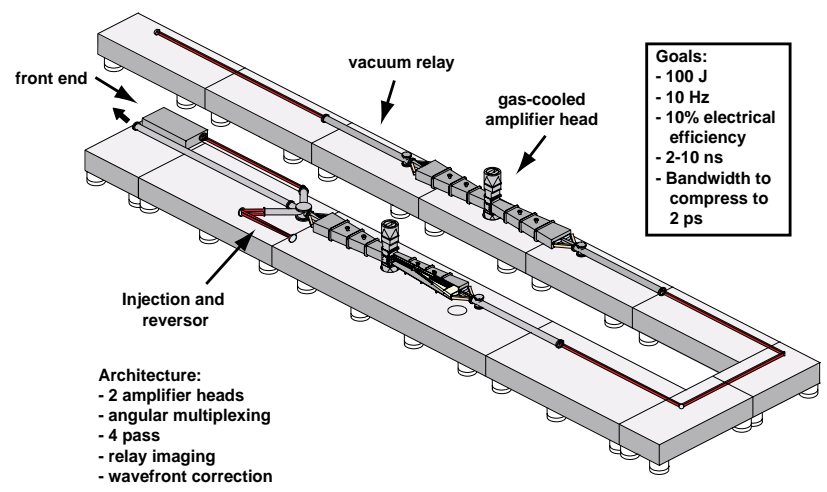

Figure 1: The diode-pumped solid state Mercury laser is a high pulse rate, next generation laser fusion driver.

\section{LASER ARCHITECTURE}

The NLC bunch structure for the $\gamma \gamma$ option consists of trains of 95 bunches separated by $2.8 \mathrm{~ns}$, with 120 trains / 
second. Each one of these must be met with a laser pulse of 1 Joule in $1.8 \mathrm{ps}$. This means that the laser architecture must be able to produce $10 \mathrm{~kW}$ of average power composed of pulses of a terawatt peak power matched to the NLC bunch structure. Any one of these requirement can be easily achieved, but a laser system capable of satisfying all three at once challenges the limits of laser technology. The Mercury laser, being developed for a laser fusion application, is close enough to be modified to satisfy these requirements. The Mercury laser, as shown in Figure 1, is designed to produce pulses of 100 Joules with a width of 2$10 \mathrm{~ns}$ with a repetition rate of $10 \mathrm{~Hz}$. An initial low-energy picosecond pulse is chirped to produce a nanosecond pulse. Once amplified in the MERCURY the pulse can be recompressed back to the picosecond level $[4,5]$ as long as it has retained a Gaussian profile after amplification [6]. This chirped pulse amplification, invented in 1985, is the basis of all lasers that can produce terawatt peak power [7]. The single pulse of 100 Joules must then be subdivided into 100 1 Joule pulses separated by $2.8 \mathrm{~ns}$ through a system of beam splitters and optical delay lines. A set of twelve of these lasers, each running at $10 \mathrm{~Hz}$, could then be combined to provide the $120 \mathrm{~Hz}$ required for the NLC.

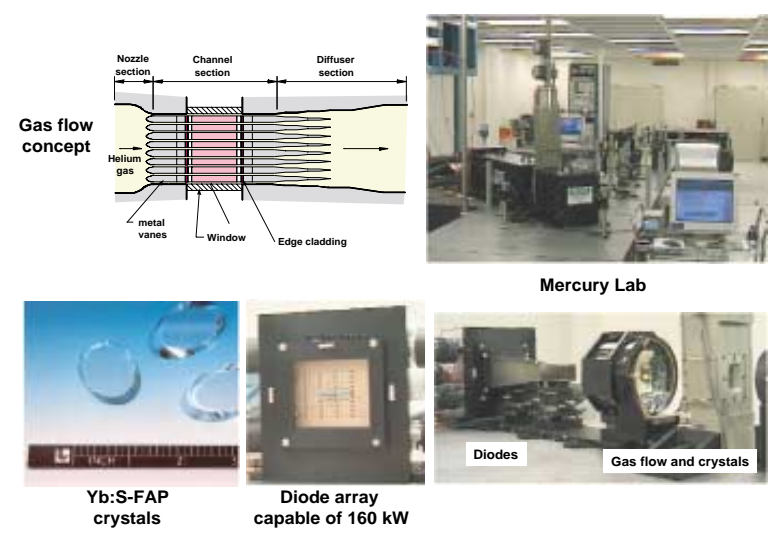

Figure 2: The Mercury laser will utilize three key technologies: gas cooling, diodes, and $\mathrm{Yb}$ :S-FAP crystals to deliver $100 \mathrm{~J}$ at $10 \mathrm{~Hz}$ with $10 \%$ efficiency.

The Mercury laser combines several key technologies, as shown in Figure 2, to achieve the required performance. The Ytterbium-Strontium-Fluorappetite (Yb:S-FAP) crystals are pumped by diode arrays. Since the diode output can be tuned to the pump band of the crystal they can achieve a $10 \%$ efficiency for converting wall plug power to laser light. The long upper state lifetime of the crystal allows the laser to be pumped more slowly, reducing the required peak diode power. This is a great advantage since the diode arrays are the cost driver for the system. The waste heat deposited in the crystal is removed by helium gas flowing perpendicular to the crystal plates. This minimizes thermal distortion and allows the laser to produce high average power while retaining good beam quality.
Previous studies of laser systems concluded that the capital cost and required power were astronomical and prohibitive. With the MERCURY architecture capital costs are around $\$ 100 \mathrm{M}$ and the required power is $200 \mathrm{~kW}$, negligible compared to the accelerator itself.

\section{OPTICS AND INTERACTION REGION}

The optics to focus the laser pulses must be co-located in the IR and they must be in the vacuum enclosure of the beam. The intensity of the laser beams requires that they be propagated in vacuum and that we keep transmissive optics to a minimum. Figure 3 shows a schematic of the interaction region with the beam pipe, masks, detector components and optics. The design starts from the standard design of the $e^{+} e^{-}$IR. The beam pipe between the IP and the masks is expanded to accommodate the mirrors and a light pipe is added outside the region of the masks to bring the light into the IR. Not shown in the figure is a complementary set of mirrors on the other side of the IP. They catch the laser pulse, reflect it and refocus it onto the opposite electron beam. Thus each laser pulse does double duty and reduces the total required laser power. The optical design is based on a four mirror telescope. The three small mirrors provide the ability to adjust the focus while the large final optic allows the beam to be focused to a near diffraction limited 10 micron diameter spot.

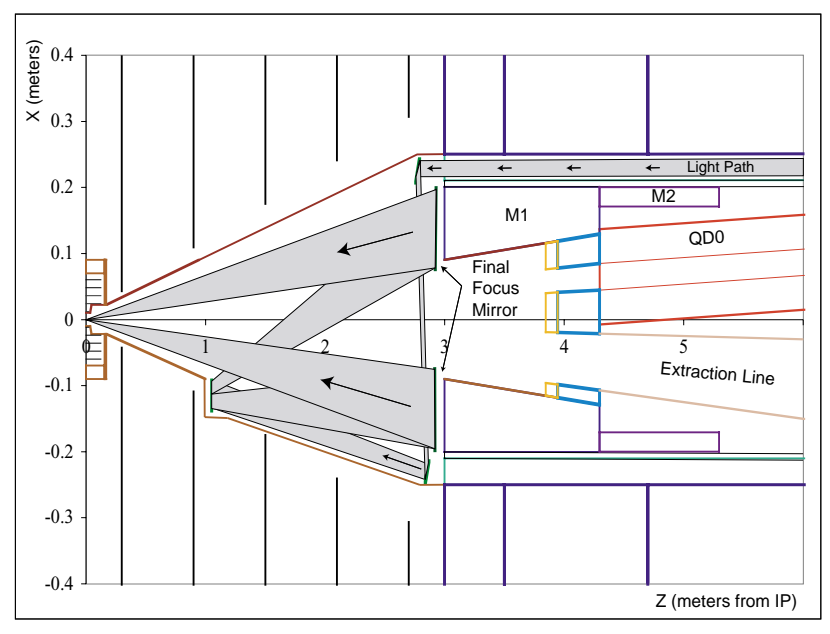

Figure 3: Optical configuration to inject the laser light into the Interaction Region. The high sub-pulse intensity requires all these optics to be reflective and mounted inside the vacuum enclosure.

A number of design choices are made to minimize the effect of the mirror's material on the accelerator and detector. The accelerator's performance is not affected since no mirror material is close to the path of the incoming and outgoing electron beams. The three small mirrors are placed off-axis and the large final focus optic is designed with a large hole in the center. This requires the laser pulse to be preshaped to be cylindrical. 

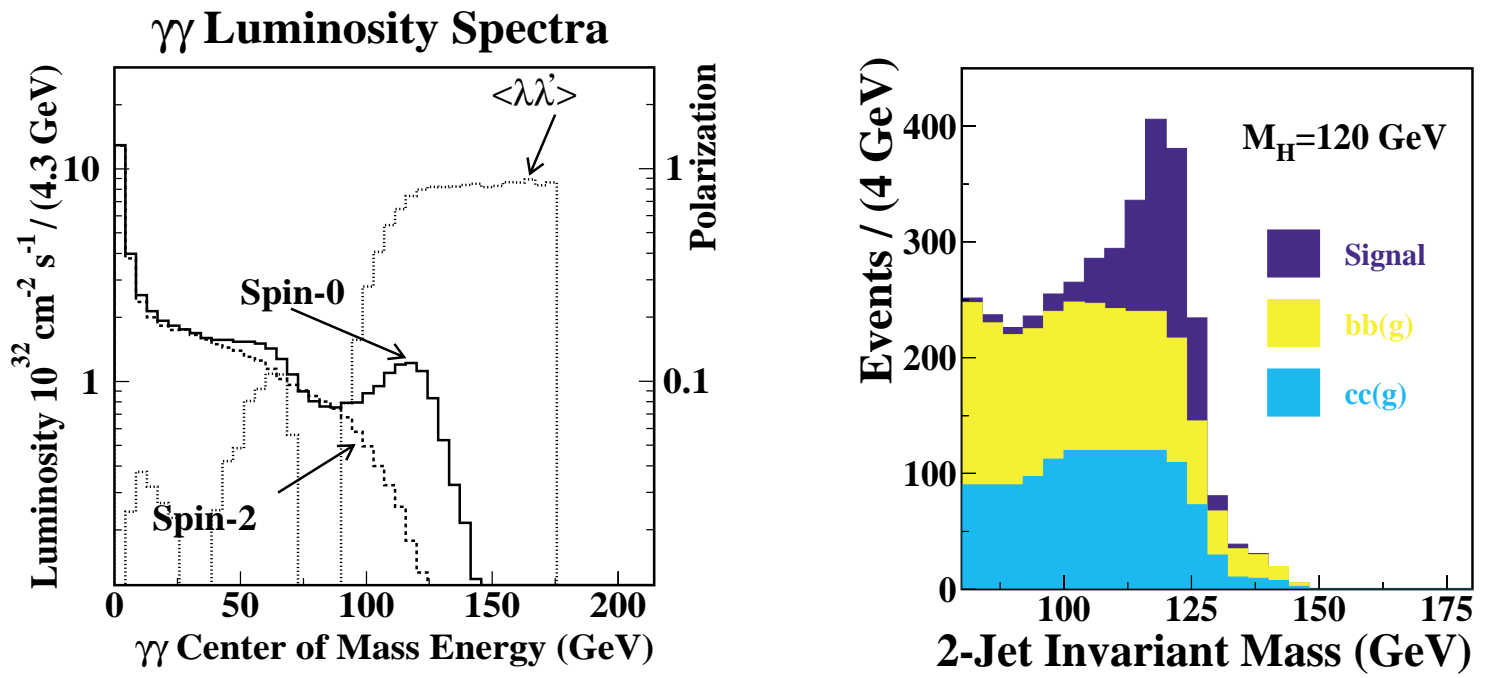

Figure 4: The luminosity spectrum for Higgs running and the 2-Jet mass distribution for the Higgs signal and the heavy quark background for a $120 \mathrm{GeV}$ Higgs mass.

The additional mirror material will degrade the low angle tracking but the effect of this is dependent on the details of any particular analysis and is unimportant for the $\gamma \gamma \rightarrow H^{0} \rightarrow b \bar{b}$ mode.

One of the main detector backgrounds in the $e^{+} e^{-}$case comes from incoherent production of $e^{+} e^{-}$pairs. The masks are designed to let these particles pass inside them before they encounter material and shower. By choosing the size of the central hole in the focusing mirror to match the size of the mask aperture we ensure a comparable background situation in the $e^{+} e^{-}$and $\gamma \gamma$ IRs.

One other modification to the interaction region is required. The extraction line aperture must be increased to \pm 10 milliradians to accommodate the increased divergence of the outgoing electron beams. In $\gamma \gamma$ collisions the electrons have a larger energy spread after the Compton backscattering and receive a larger deflection from the field of the oncoming electron bunch. To prevent interference between the extraction line and the final focus quadrupole the crossing angle is increased from 20 to 30 milliradians. The first two layers of the SVX now have a direct line of sight back to the beam dump. They see a flux of $10^{11}$ neutrons $/ \mathrm{cm}^{2} /$ year. The standard CCDs will need to be replaced with a more radiation hard technology.

\section{THE BENCHMARK MODE $\gamma \gamma \rightarrow H^{0} \rightarrow B \bar{B}$.}

The measurement of the two photon width of the Higgs is one of the main physics analyses for a photon collider. Since $\gamma \gamma \rightarrow H$ proceeds through a loop diagram and all particles with mass and charge can contribute to it, this mode is an excellent probe of new physics beyond the standard model. Using our design for the laser and optics we have used the CAIN program to simulate the $\gamma \gamma$ luminosity spectrum. Figure 4 shows the calculated luminosity for running conditions optimized for a $120 \mathrm{GeV}$ Higgs. The high energy peak is dominated by photons from primary Compton backscatters. The low energy tail comes from photons produced when spent electrons multiply interact with the laser and from beamstrahlung photons. Control of the laser and electron polarization allow spin- $0 \gamma \gamma$ states to be selected over spin-2 states. This enhances the $\gamma \gamma \rightarrow H$ signal and suppresses the $\gamma \gamma \rightarrow b \bar{b}, c \bar{c}$ backgrounds. A Monte Carlo study [8] of the $\gamma \gamma \rightarrow H \rightarrow b \bar{b}$ mode has shown that the rate $\Gamma(\gamma \gamma \rightarrow H \rightarrow b \bar{b})$ can be measured to an accuracy of $5 \%$ with one year's luminosity. Additionally, by producing linearly polarized laser photons, $\gamma \gamma$ states of definite $\mathrm{CP}$ can be produced. This allows the $\mathrm{CP}$ nature of the Higgs to be probed with an accuracy of $20 \%$ for one year's running.

\section{REFERENCES}

[1] I. Ginzburg, G. Kotkin, V.Serbo and V. Telnov, Pizma ZhETF 34 (1981) 514; JETP Lett. 34 (1982) 491 (Preprint INF 81-50, Novosibirsk (1981) in English).

[2] V. Telnov, Nucl. Instr. and Meth. A 355 (1995) 3.

[3] K. Yokoya, "A Computer Simulation Code for the BeamBeam Interaction in Linear Colliders", KEK report 85-9, Oct. 1985.

[4] D. Strickland and G. Mourou, "Compression of amplified chirped optical pulses," Opt. Commun. 56, 219-221 (1985).

[5] E.B. Treacy, "Optical pulse compression with diffraction gratings,” IEEE J.Quantum Electron. QE-5, 454-458 (1969).

[6] M.D. Perry, F.G. Patterson, and J. Weston, "Spectral shaping in chirped-pulse amplification," Opt. Lett. 15, 381-383 (1990)

[7] M.D. Perry and G. Mourou, "Terawatt to petawatt subpicosecond lasers," Science 264, 917-924 (1994).

[8] D. Asner, J. Gronberg, J. Gunion, T. Hill, UCRL-ID-143967. 